\title{
A Vilsmeier Chloroformylation by Continuous
}

\section{Flow Chemistry}

Manuel Carrera, ${ }^{1,+}$ Laurens De Coen, ${ }^{1,+}$ Michelle Coppens ${ }^{2}$, Wim Dermaut ${ }^{2}$

and Christian V. Stevens ${ }^{1, *}$

${ }^{1}$ Department of Green Chemistry and Technology, Faculty of Bioscience Engineering, Ghent University, Campus Coupure, Coupure Links 653, B-9000 Ghent, Belgium

${ }^{2}$ AGFA-GEVAERT NV, Septestraat 27, B-2640 Mortsel, Belgium 


\section{General:}

Commercially available starting materials (Sigma-Aldrich, Fisher Scientific, TCI Europe) and solvents were used without further purification. NMR spectra were recorded on a Bruker Avance III (400 MHz for ${ }^{1} \mathrm{H}-\mathrm{NMR}, 101 \mathrm{MHz}$ for ${ }^{13} \mathrm{C}-\mathrm{NMR}$ and $162 \mathrm{MHz}$ for ${ }^{31} \mathrm{P}-\mathrm{NMR}$ ), equipped with a ${ }^{1} \mathrm{H} / \mathrm{BB}$ z-gradient probe $\left(\mathrm{BBO}, 5 \mathrm{~mm}\right.$ ). DMSO- $\mathrm{d}_{6}$ and $\mathrm{CDCl}_{3}$ were used as solvents and TMS as internal chemical shift standard in the $\mathrm{CDCl}_{3}$. LC-MS analysis was performed with Agilent 1200 Series HPLC equipped with a Supelco Ascentis Express C18 column (3 cm x $4.6 \mathrm{~mm}, 2.7 \mu \mathrm{m}$ fused-core particles, $90 \AA$ ), Phenomenex Guard column (SecurityGuard Standard) and a UV-DAD detector. MS spectra were obtained with an Agilent 1100 Series MS with electrospray ionisation $(70 \mathrm{eV})$ with a single quadrupole detector. IR spectra were obtained from neat samples with a Quest ATR accessory with diamond crystal puck using a Shimadzu IRAFFINITY-1S Fourier Transform Infrared Spectrophotometer. HRMS was recorded using a Thermo Fisher QExtractive MS, with loop injection in 350 $\mu \mathrm{L} / \mathrm{min}$. $\mathrm{MeOH}+0.1 \% \mathrm{FA} / \mathrm{H}_{2} \mathrm{O}+0.1 \% \mathrm{FA} 50 / 50$ and ESI positive mode (3.5 $\mathrm{kV}$ of Spray Voltage) with a mass resolution of 70000 (FWHM).

Flow synthesis of compound 1 (representative example, Table 3, Entry1):

The synthesis of compound $\mathbf{1}$ was performed in the flow reaction set-up as described above. The internal volume of reactors $\mathrm{R} 1=8 \mathrm{~mL}, \mathrm{R} 2=19.6 \mathrm{~mL}, \mathrm{R} 3=14 \mathrm{~mL}$. $\mathrm{POCl}_{3}$ was pumped at $0.22 \mathrm{~mL} / \mathrm{min}, \mathrm{NFM}$ was pumped at $0.63 \mathrm{~mL} / \mathrm{min}$ and cyclohexanone was pumped at 0.08 $\mathrm{mL} / \mathrm{min}$. The aqueous sodium acetate solution was prepared by dissolving $123 \mathrm{~g}$ of sodium acetate trihydrate in $450 \mathrm{~g}$ of water. This solution was pumped at $3.72 \mathrm{~mL} / \mathrm{min}$. The thermostatic oil bath was kept at $90{ }^{\circ} \mathrm{C}$. After an equilibration period of 105 minutes, a sample was collected for 25 minutes. The sample was collected in an Erlenmeyer flask equipped with a stirring bar and cooled with an ice bath. After stirring for 30 minutes at $0{ }^{\circ} \mathrm{C}$, the product was 
collected by filtration over a glass sintered filter and rinsed two times with $10 \mathrm{~mL}$ of ice-cold water. The filtered solids were transferred to a glass round-bottomed flask and dried under high vacuum to afford compound $\mathbf{1}$ as a yellow powder $(2.643 \mathrm{~g}, 79 \%)$.

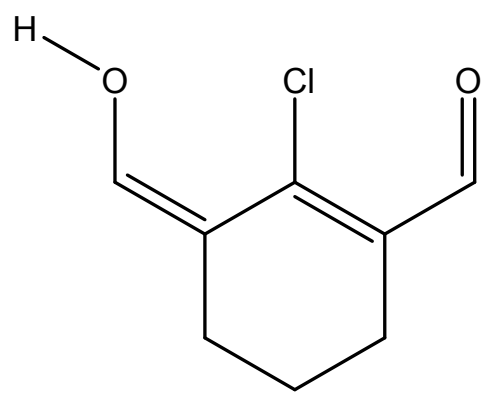

2-Chloro-1-formyl-3-(hydroxymethylene)cyclohex-1-ene (1)

${ }^{1} \mathrm{H}-\mathrm{NMR}\left(400 \mathrm{MHz}, \mathrm{CDCl}_{3}\right.$ ): $\delta 1.72$ (quint, $\left.J=6.2 \mathrm{~Hz}, 2 \mathrm{H}\right), 2.43(\mathrm{t}, J=6.1 \mathrm{~Hz}, 4 \mathrm{H}), 2.49(\mathrm{t}, J=$ $5.7 \mathrm{~Hz}, 2 \mathrm{H}), 5.40(\mathrm{~d}, J=7.8 \mathrm{~Hz}, 1 \mathrm{H}), 7.51(\mathrm{~d}, J=7.0 \mathrm{~Hz}, 1 \mathrm{H}), 10.24(\mathrm{~s}, 1 \mathrm{H}) \mathrm{ppm}$.

${ }^{1} \mathrm{H}-\mathrm{NMR}$ (400 MHz, DMSO-d6): $\delta 1.58$ (quint, $\left.J=6.1 \mathrm{~Hz}, 2 \mathrm{H}\right), 2.36$ (t, $J=5.9 \mathrm{~Hz}, 4 \mathrm{H}$ ), 8.89 (s, 1H), $10.84(\mathrm{~s}, 1 \mathrm{H}) \mathrm{ppm}$.

${ }^{13} \mathrm{C}-\mathrm{NMR}$ (101 MHz, DMSO-d6): $\delta 20.41,24.15,146.51 \mathrm{ppm}$.

IR (ATR, cm-1): 538, 638, 708, 735, 997, 1180, 1387, 1454, 1597, 1796, 2953, 3152.

MS (ESI): m/z (\%) 173.0/175.0 (100/34, [M+H] $\left.]^{+}\right), 174.0(11)$.

HRMS (ESI): calc. for $\left[\mathrm{C}_{8} \mathrm{H}_{10} \mathrm{ClO}_{2}+\mathrm{H}\right]^{+}$173,0369, found 173, 0366 .

m.p. $=138^{\circ} \mathrm{C}\left(\text { lit. } 130-131^{\circ} \mathrm{C}\right)^{1}$ 
${ }^{1} \mathrm{H}-\mathrm{NMR}\left(400 \mathrm{MHz}, \mathrm{CDCl}_{3}\right.$ ) compound 1.

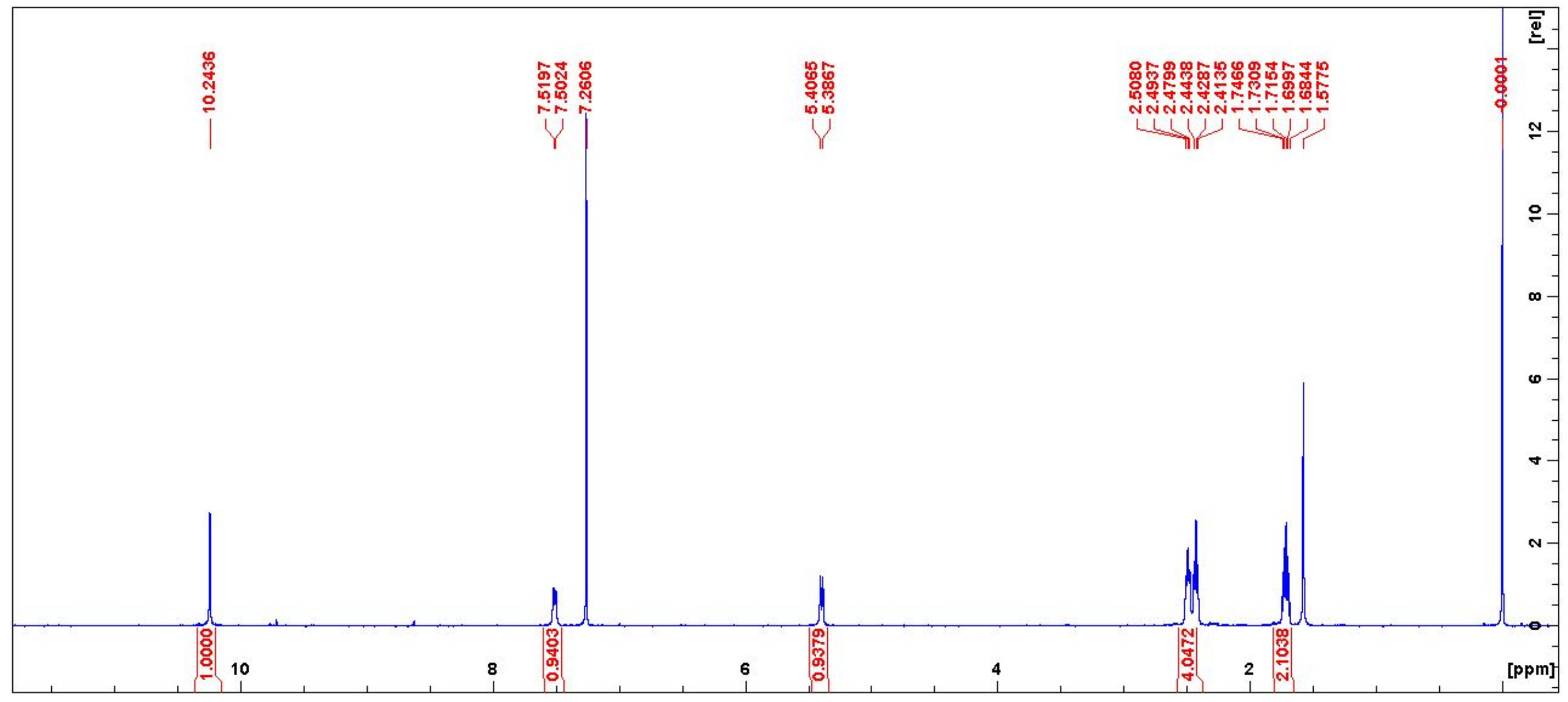


${ }^{1} \mathrm{H}-\mathrm{NMR}$ (400 MHz, DMSO-d6) compound 1.

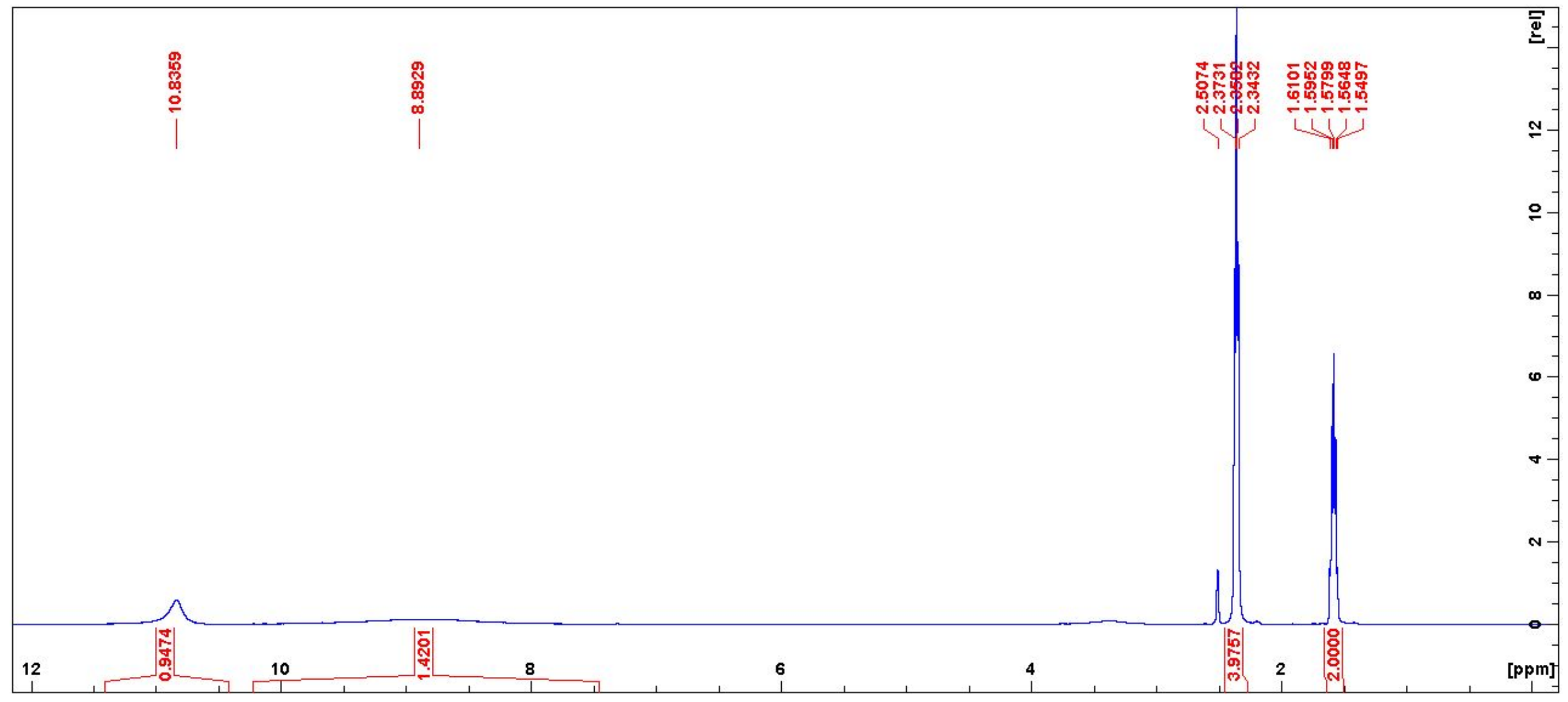


${ }^{13} \mathrm{C}-\mathrm{NMR}$ (101 MHz, DMSO-d6) compound 1.

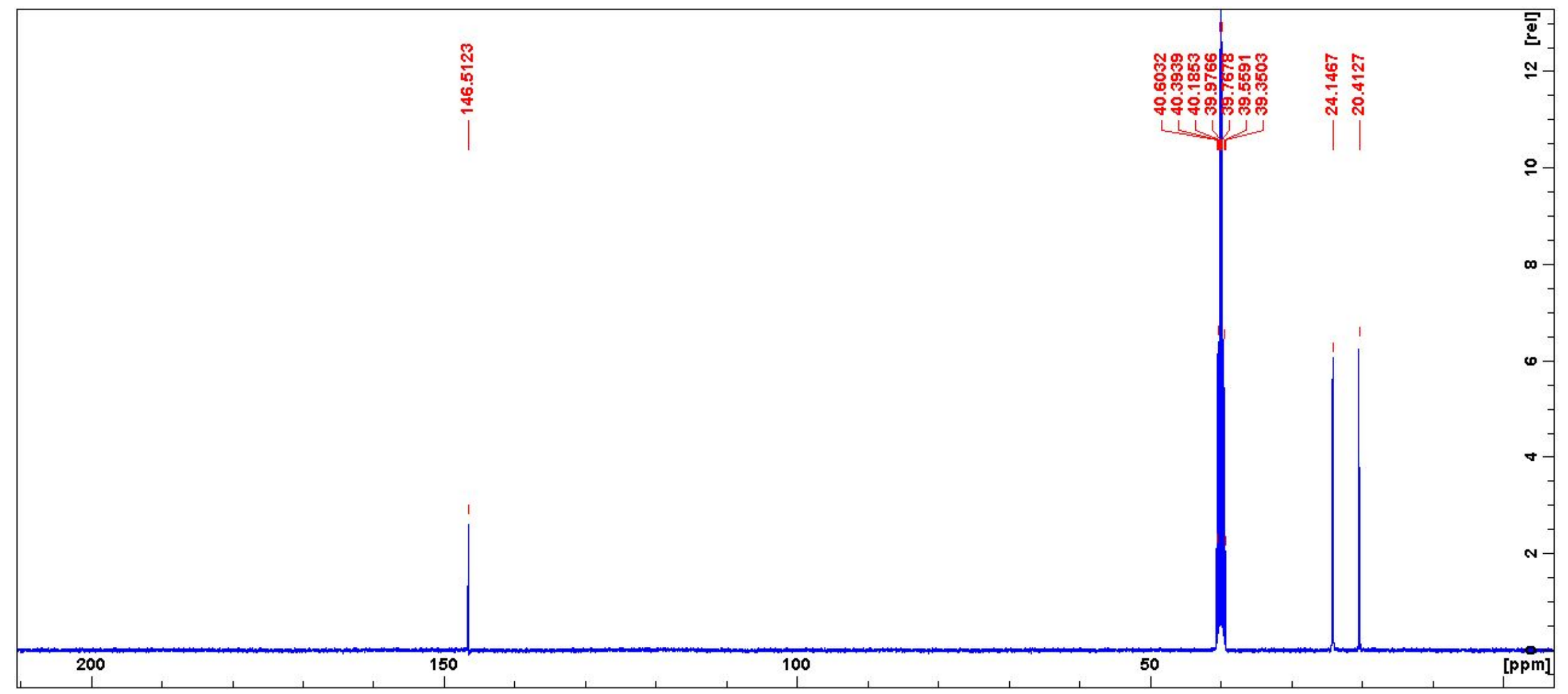


IR (neat)

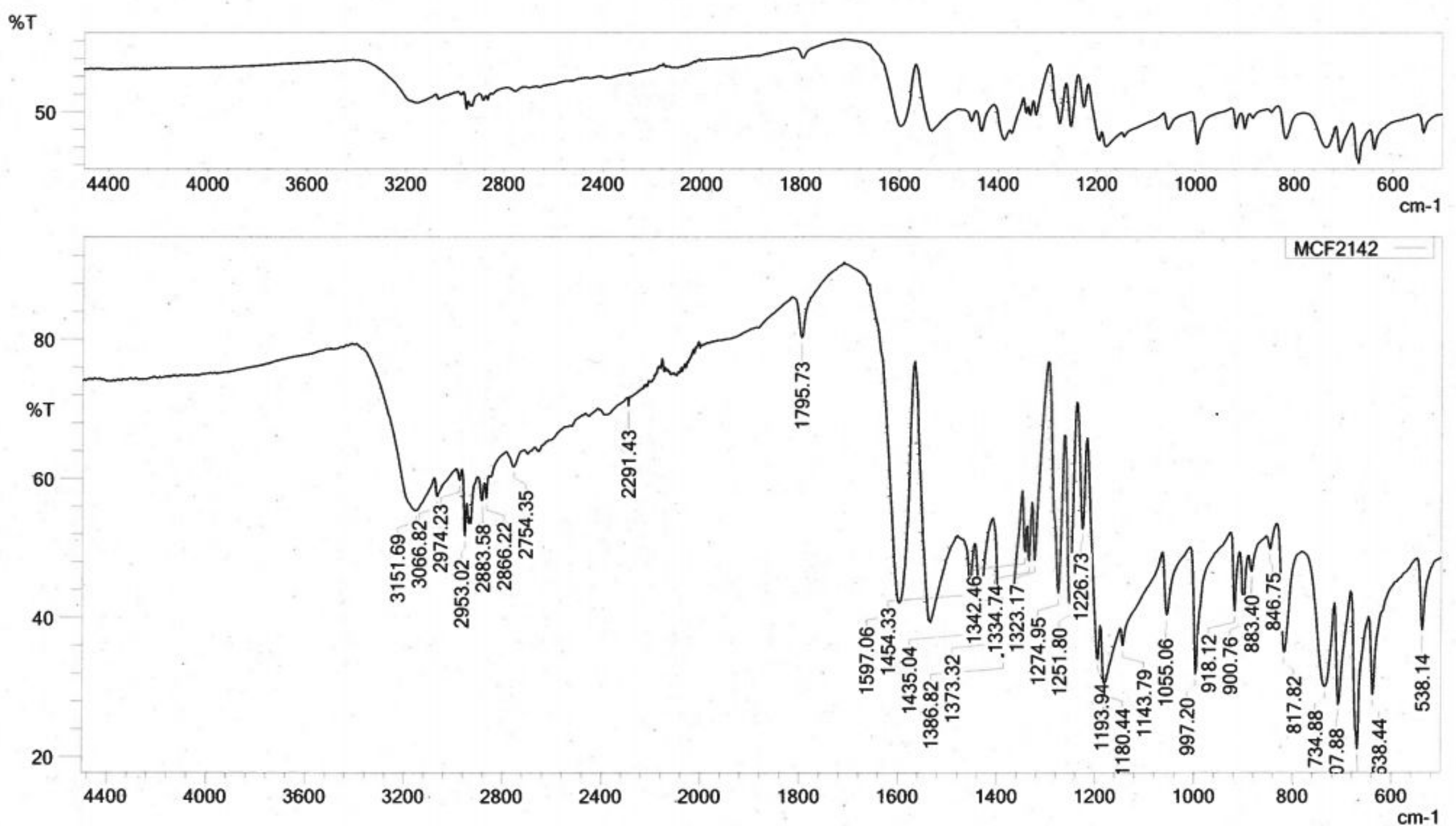


HRMS (ESI)

E:IDatalqe46532

Manuel Carrera

07/02/20 10:19:30

MCF217

रT: $0.01-2.00$ SM: 7B

NL:
1.80E10

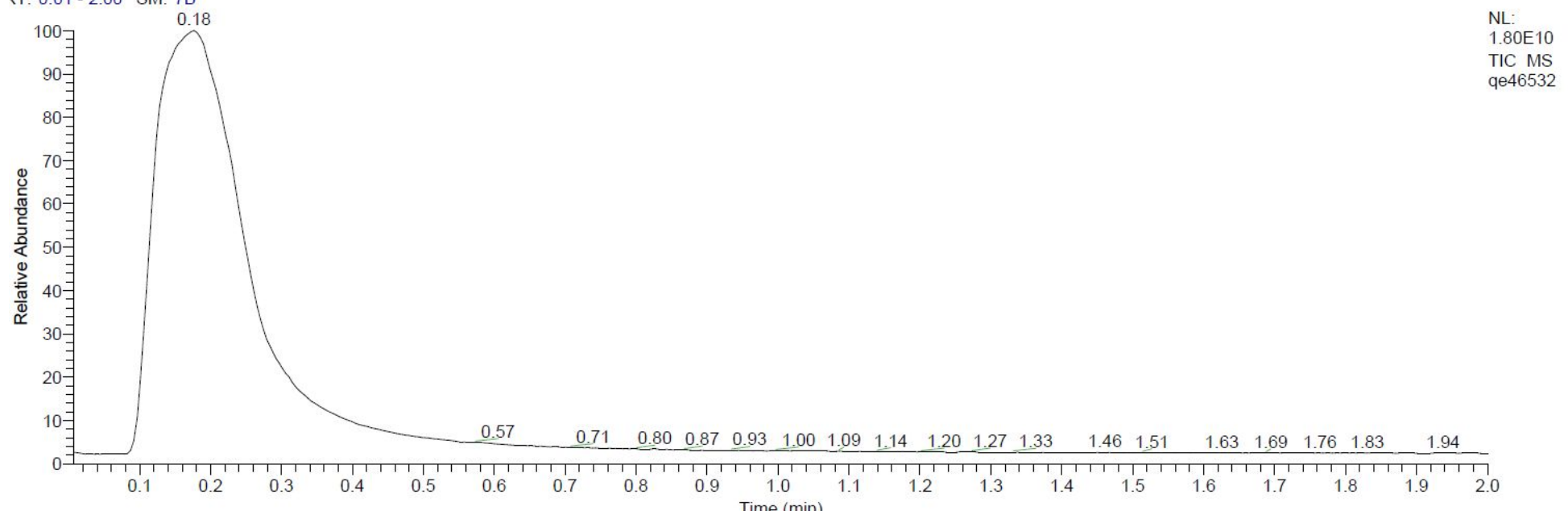

le46532 \#29-55 RT: 0.13-0.24 AV: 27 NL: 9.39E9

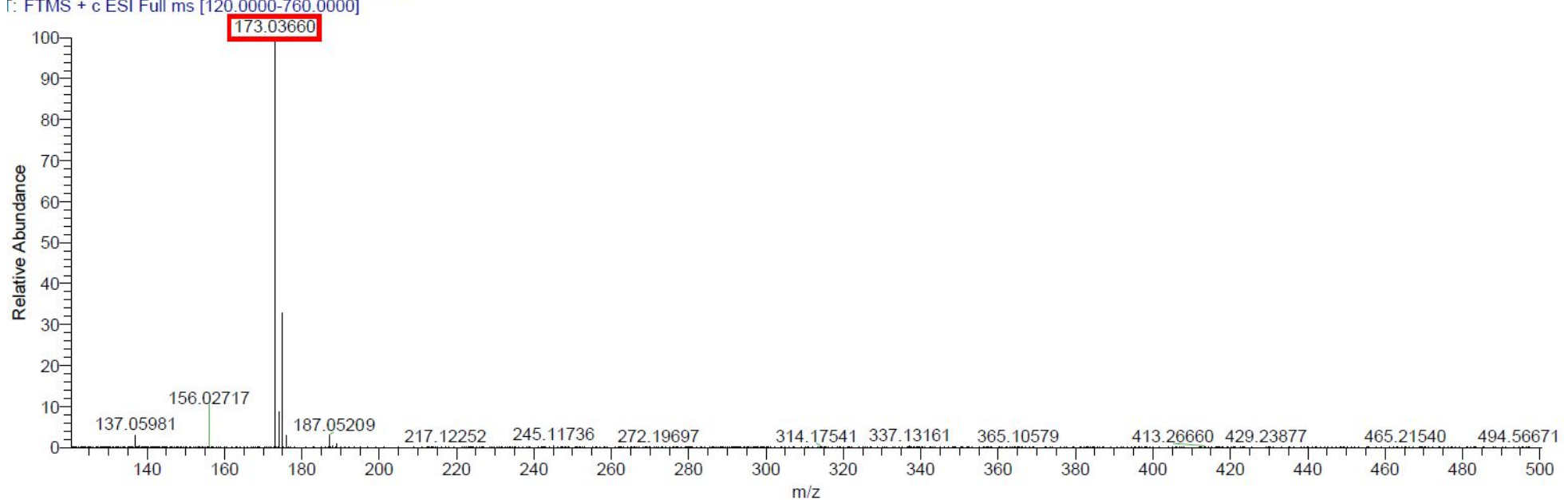




\section{REFERENCES}

1) Reynolds, G.A.; Drexhage, K.H. Stable heptamethine pyrylium dyes that absorb in the infrared. J. Org. Chem. 1977, 42, 855-888. 